\title{
Electronic properties of silicene in BN/silicene van der Waals heterostructures*
}

\author{
Ze-Bin Wu (吴泽宾), Yu-Yang Zhang (张余洋), Geng Li (李更), \\ Shixuan Du (杜世萱) ${ }^{\dagger}$ and Hong-Jun Gao (高鸿钧)
}

Institute of Physics \& University of Chinese Academy of Sciences, Chinese Academy of Sciences, Beijing 100190, China

Key Laboratory of Vacuum Physics, Chinese Academy of Sciences, Beijing 100190, China

Silicene is a promising 2D Dirac material as building block of van der Waals heterostructures (vdWHs). Here we investigate the electronic properties of hexagonal boron nitride/silicene (BN/Si) vdWHs using first-principles calculations. We calculate the energy band structures of $\mathrm{BN} / \mathrm{Si} / \mathrm{BN}$ heterostructure with different rotation angles and find the electronic properties of silicene is retained and protected robustly by $\mathrm{BN}$ layers. In $\mathrm{BN} / \mathrm{Si} / \mathrm{BN} / \mathrm{Si} / \mathrm{BN}$ heterostructure, we find the band structure near the Fermi energy is sensitive to the stacking configurations of the silicene layers due to interlayer coupling. The coupling is reduced by increasing the number of $\mathrm{BN}$ layers between silicene layers and become negligible in $\mathrm{BN} / \mathrm{Si} /(\mathrm{BN})_{3} / \mathrm{Si} / \mathrm{BN}$. In $(\mathrm{BN})_{\mathrm{n}} / \mathrm{Si}$ superlattices, the band structure undergoes a conversion between Dirac lines and Dirac points with different number of BN layers between silicene layers. Calculations of silicene sandwiched by other 2D materials reveal that silicene sandwiched by low-carbon-doped boron nitride or $\mathrm{HfO}_{2}$ is semiconducting.

Keywords: silicene, BN, electronic property, heterostructure

* Project supported by the National Key Research and Development Program of China (No. 2016YFA0202300), the National Natural Science Foundation of China (Nos. 61390501, 61471337), the National Basic Research Program of China (No.

2013CBA01600), the CAS Pioneer Hundred Talents Program, and the Beijing Nova Program (No. Z181100006218023).

Corresponding author. E-mail: sxdu@iphy.ac.cn 


\section{Introduction}

Since the successful exfoliation of graphene in $2004,{ }^{[1,2]}$ more than a hundred intriguing 2D materials have been explored. ${ }^{[3-6]}$ However, only a few of them are theoretically predicted to be Dirac materials. ${ }^{[7]}$ The existence of Dirac cones in 2D materials gives rise to many novel physical properties, such as half-integer/fractional quantum Hall effect, ${ }^{[2,8-10]}$ ultrahigh carrier mobility, ${ }^{[11]}$ etc. ${ }^{[12]}$ According to firstprinciples calculations, ${ }^{[13,14]}$ monolayer silicene is a Dirac material with a stable buckled honeycomb structure. ${ }^{[15]}$ The Fermi velocity of silicene is predicted to be $\sim 10^{6}$ $\mathrm{m} / \mathrm{s}$, and its intrinsic carrier mobility has the same order of magnitude as that of graphene. ${ }^{[16]}$ Besides, the electronic properties of silicene have a better tunability than those of graphene. ${ }^{[17-22]}$ These novel properties together with the possibly inherent compatibility with traditional silicon-based nanotechnology make silicene a promising candidate for electronic devices and a distinct building block for van der Waals layered materials.

Silicene has been grown on several substrates, such as $\operatorname{Ag}(111),{ }^{[23-26]} \operatorname{Ir}(111),{ }^{[27,28]}$ $\mathrm{Ru}(0001),{ }^{[29]}$ and $\mathrm{ZrB}_{2}(0001) \cdot{ }^{[30]}$ Transistors have also been fabricated with epitaxial silicene on $\operatorname{Ag}(111)$ thin film. ${ }^{[31]}$ However, there is no convincing experimental evidence supporting the existence of Dirac cones in epitaxial silicene because of the strong coupling with these substrates. ${ }^{[27,29,30,32-37]}$ Silicene's surface is indeed very active because of the dangling Si bonds. The exposed silicene interacts easily with other active materials so that the electronic properties can be modified easily in unexpected ways. However, silicene has been found to interact weakly with graphene ${ }^{[38]}$ and hexagonal boron nitride $(\mathrm{BN})^{[17,39]}$. Dirac cones of silicene shift above the Fermi level when sandwiched by graphene. In contrast, BN does not cause the shift of Dirac cones. Therefore, BN-protected silicene could be a promising block for building van der Waals layered heterostructures (vdWHs). On the other hand, silicene sandwiched between some other 2D materials can be semiconducting, which is promising for applications in electronic devices. ${ }^{[40]}$ Similar as the cases of molecules encapsulated by nanotubes or graphene layers, silicene is also energetically stabilized by the cladding layers. ${ }^{[41]}$

In this paper, we investigate electronic structures of BN-protected silicene in heterostructures and superlattices by first-principles calculations. Using "Si" to denote silicene, first we calculated the properties of $\mathrm{BN} / \mathrm{Si} / \mathrm{BN}$ heterostructures with different 
rotation angels and lateral shifts. Results show that rotation, lateral shift, and limited strain in silicene will not cause gap opening. To take advantage of the ultra-high carrier mobility of silicene, we propose a $\mathrm{BN} / \mathrm{Si} / \mathrm{BN} / \mathrm{Si} / \mathrm{BN}$ heterostructures, where each silicene layer is a conducting channel. We calculated the electronic properties of it and find that the band structure near the Fermi energy is sensitive to different stacking configurations of silicene layers due to interlayer coupling between silicene layers. We find that the coupling becomes negligible when silicene layers are separated by three $\mathrm{BN}$ layers in $\mathrm{BN} / \mathrm{Si} /(\mathrm{BN})_{\mathrm{n}} / \mathrm{Si} / \mathrm{BN}$ heterostructure. We also calculated potential $(\mathrm{BN})_{\mathrm{n}} / \mathrm{Si}$ superlattices, and find that a conversion between Dirac points to Dirac lines can be achieved by changing the number of BN layers between silicene layers. Moreover, we calculated the band structures of silicene sandwiched by low-carbon-doped BN (1$\mathrm{BCN}$ ), high-carbon-doped $\mathrm{BN}(\mathrm{h}-\mathrm{BCN})$, and $\mathrm{HfO}_{2}$ and graphene/Si/BN. We find that the Dirac cones of silicene are opened in all of these heterostructures, but only 1$\mathrm{BCN} / \mathrm{Si} / \mathrm{l}-\mathrm{BCN}$ and $\mathrm{HfO}_{2} / \mathrm{Si} / \mathrm{HfO}_{2}$ are semiconducting with band gaps of $19 \mathrm{meV}$ and $131 \mathrm{meV}$ respectively.

\section{Methods}

All quantum-mechanical calculations were carried out based on the density functional theory (DFT) as implemented in the Vienna Ab-initio Simulation Package (VASP). ${ }^{[42-44]}$ Electron-ion interactions were represented by projected augmented wave (PAW) potentials. ${ }^{[45]}$ Exchange-correlation interactions were treated within the generalized gradient approximation (GGA-PBE) ${ }^{[46]}$ The method of DFT-D3 to include van der Waals interactions was used in all the calculations of heterostructures. ${ }^{[47]}$ The wave functions were expanded using a plane-wave basis set with an energy cutoff of $520 \mathrm{eV}$. For 2D structures, a vacuum layer larger than $20 \AA$ was used to avoid interactions between layers in neighboring supercells. The force on each atom is relaxed to less than $0.01 \mathrm{eV} / \AA$. The Brillouin zone was sampled using a $\Gamma$-centered $18 \times 18$ mesh for $(2 \times 2)$ silicene in self-consistent calculations.

\section{Results and discussions}

\subsection{Electronic properties of $\mathrm{BN} / \mathrm{Si} / \mathrm{BN}$}


It has been reported that the Dirac cone of silicene is protected when a $(2 \times 2)$ silicene is sandwiched by two $(3 \times 3)$ BN layers. ${ }^{[17]}$ While in the real fabricating process, there are always lattice mismatches, rotation angles (misalignment), strains, and so on. To investigate the effects of above-mentioned situations in heterostructures, we build different $\mathrm{BN} / \mathrm{Si} / \mathrm{BN}$ supercells with different stacking configurations and calculate the energy band structures. The results are presented in table 1 .

To eliminate the effect of strain in silicene in these heterostructures, the lattice constants of silicene is kept the same as that of its free-standing states and the heterostructures are otherwise fully relaxed. For all the $\mathrm{BN} / \mathrm{Si} / \mathrm{BN}$ heterostructures shown in Table 1 , the calculated binding energy is $32 \mathrm{meV} / \AA^{2}$. Compared with the binding energies for bilayer graphene which are $40 \mathrm{meV} / \AA^{2}$ for A-A stacking and 42 $\mathrm{meV} / \AA^{2}$ for A-B stacking, the binding between $\mathrm{BN}$ and silicene is weaker. The binding energy is defined as $E_{\text {Binding }}=\left(2 \times E_{B N}+E_{S i}-E_{\text {Heterostructure }}\right) /(2 \times$ area $)$ for $\mathrm{BN} / \mathrm{Si} / \mathrm{BN}$ and $E_{\text {Binding }}=\left(2 \times E_{\text {Graphene }}-E_{\text {Bilayer-graphene }}\right) /$ area for bilayer graphene. The negative

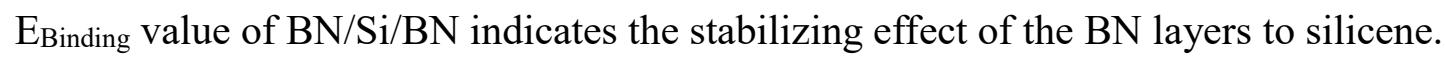

As a result of the small binding energy, there are probably different rotation angles in $\mathrm{BN} / \mathrm{Si}$ heterostructures in the fabrication process in experiments. The calculations show that the electronic properties are nearly not affected by the rotation angles for $\mathrm{BN} / \mathrm{Si}$ heterostructures due to weak van der Waals interaction. We also investigate the impact of lateral shifts in $\mathrm{BN} / \mathrm{Si} / \mathrm{BN}$. Calculations show that lateral shifts almost have no effect on both the electronic properties and the total energy. Considering the possible strain in silicene layer induced by lattice mismatches in heterostructures, we calculate the energy band structure of silicene under strain of less than 3\%. Calculation results show that the Dirac cones are not opened up and the electronic properties are nearly not affected by the strain. Moreover, the possible lattice distortion or rumpling induced by local strain would only have an effect on the local electronic properties. Therefore, we conclude that the electronic properties of silicene are protected robustly when sandwiched between BN layers.

\subsection{Electronic properties of multilayer $\mathrm{BN} / \mathrm{Si} / \mathrm{BN}$}

Since silicene is left intact when sandwiched between BN layers under different rotation angles, lateral shifts, and limited strains in silicene, we use $\mathrm{BN} / \mathrm{Si} / \mathrm{BN}$ as a 
building block for multilayer heterostructures. To take advantage of the ultra-high carrier mobility of silicene, we propose a multilayer BN/Si heterostructures, where each silicene layer is a conducting channel. We first discuss potential $\mathrm{BN} / \mathrm{Si} / \mathrm{BN} / \mathrm{Si} / \mathrm{BN}$ heterostructures, in which two silicene layers are positioned in an A-A stacking configuration. The structures and calculation results of $\mathrm{BN} / \mathrm{Si} / \mathrm{BN} / \mathrm{Si} / \mathrm{BN}$ are shown in Fig. 1(a). As seen from the energy bands shown in Fig. 1(b), there are two sets of Dirac cones above and below the Fermi energy, marked by grey circles. The shift of these two sets of Dirac cones indicates that there is coupling between those two silicene layers. The energy separation of these two sets of Dirac cones is $143 \mathrm{meV}$. Because of the shift of Dirac cones from the Fermi level, there are crosses between the energy bands as shown in the energy band structures. As a result, a small gap of around $4 \mathrm{meV}$ is opened due to perturbations at the Fermi level.

The distance between two silicene layers in $\mathrm{BN} / \mathrm{Si} / \mathrm{BN} / \mathrm{Si} / \mathrm{BN}$ is $7.6 \AA$. When two silicene layers are separated by a vacuum layer of $7.6 \AA$, there is nearly no interaction between them and the Dirac cones of them are overlapped. On the other hand, the energy states of $\mathrm{BN} / \mathrm{Si} / \mathrm{BN} / \mathrm{Si} / \mathrm{BN}$ near the Fermi level $\left(\mathrm{E}_{\mathrm{f}} \pm 0.3 \mathrm{eV}\right)$ are contributed by both silicene and BN layers, as seen from the partial charge density in Fig. 1(c). Therefore, we conclude that the interlayer coupling between silicene layers is mediated by the $\mathrm{BN}$ layer between them. According to this result, a question arises that whether more BN layers between silicene layers decrease the coupling or not.

To further investigate the interlayer coupling and its impact on the electronic properties, we increase the number of BN layers between silicene layers. The energy band structures of the $\mathrm{BN} / \mathrm{Si} /(\mathrm{BN})_{n} / \mathrm{Si} / \mathrm{BN}$ and $\mathrm{BN} / \mathrm{Si} /(\mathrm{BN})_{n} / \mathrm{Si} / \mathrm{BN}$ structures are shown in Figs. 1(d) and 1(e), respectively. As seen in Fig. 1(d), the energy difference between the two sets of Dirac cones reduces to $18 \mathrm{meV}$, indicating that the interlayer coupling is weakened by increasing the number of BN layers. The shift of the Dirac cones becomes negligible when the two silicene layers are separated by three BN layers, as shown in Fig. 1(e). In short, the interaction between silicene layers is weakened by increasing the number of BN layers. Therefore, the proposed multi-conducting-channel $\mathrm{BN} / \mathrm{Si}$ heterostructure is only possible when silicene layers are separated by three or more than three BN layers.

In the heterostructures mentioned above, only A-A stacking silicene layers are 
considered. Because of the weak binding between $\mathrm{BN}$ and silicene in $\mathrm{BN} / \mathrm{Si}$ heterostructures, there can be different sequences for silicene layers during fabrication in experiments. We investigate the impact of different stacking configurations of those two silicene layers resembling bilayer graphene. For graphene, there are two common stacking configurations, A-A stacking and A-B stacking. Different from graphene, there are five different high-symmetry stacking configurations for two silicene layers due to its buckled structure. The heterostructures and the energy band structures near the Fermi level are shown in Fig. 2. The results of A-A stacking configurations are shown in Fig. 2(a) and 2(b) and the results of A-B stacking configuration are shown in Fig. 2(c)-(e). The energy band structure of an A-B stacking configuration is close to each other whereby the Dirac cones are destroyed, with a negligible gap appearing at K points. In an A-A stacking configuration, the Dirac cones are preserved but shifted to different sides of the Fermi level as discussed before. For A-A stacking configuration in mirror symmetry, the Dirac cones are destroyed. The total binding energy of BN/Si/BN/Si/BN is almost the same of around $116 \mathrm{meV} / \AA^{2}$. (The binding energy are calculated as $E_{\text {Binding }}$ $=\left(3 \times \mathrm{E}_{\mathrm{BN}}+2 \times \mathrm{E}_{\mathrm{Si}}-\mathrm{E}_{\text {Heterostructure }}\right) /$ area. $)$

\subsection{Electronic properties of BN/Si superlattices}

We further investigated $(\mathrm{BN})_{\mathrm{n}} /$ silicene superlattices, in which silicene layers are positioned in A-A configuration, with $\mathrm{n}=1,2,3$. The unit cell and the first Brillouin zone are shown in Fig. 3(a). Band structures of the superlattices are shown in Fig. 3(b)(d). Consistent with the results above, the Dirac cones shift from the Fermi level when silicene layers are separated by less than three BN layers, forming Dirac lines along the K-H lines in the reciprocal space as seen in Fig 3(b) and 3(c). When the number of BN layers is increased to three, the Dirac cones return to the positions of $E_{f} \pm 2 \mathrm{meV}$, indicating that the coupling is negligible between silicene layers and quasi-freestanding silicene is achieved in this van der Waals superlattice. T. P. Kaloni et al ${ }^{[48]}$ reported that quasi-free-standing silicene can be achieved in $\mathrm{Si} / \mathrm{BN}$ superlattice where adjacent silicene layers are separated by only one layer of BN. However, according to our result, one layer of $\mathrm{BN}$ is not enough to isolate silicene layers in $\mathrm{BN} / \mathrm{Si}$ superlattice and there are Dirac lines but not Dirac points. Based on the results, it is safer to use at least three BN layers as cladding layers at each side of silicene to protect the electronic properties from being modified. 


\subsection{Semiconducting silicene in heterostructures}

It is important to open a gap in silicene for its application in electronic devices. Many different ways based on theoretical calculations have been reported to achieve this goal. ${ }^{[17-22]}$ Here we present the results that silicene sandwiched by $1-\mathrm{BCN}$ or $\mathrm{HfO}_{2}$ is semiconducting. We calculated the band structures of $\mathrm{Gr} / \mathrm{Si} / \mathrm{BN}, \mathrm{BCN} / \mathrm{Si} / \mathrm{BCN}$, and $\mathrm{HfO}_{2} / \mathrm{Si} / \mathrm{HfO} 2$ heterostructures. $\mathrm{BCN}$ can be fabricated from either $\mathrm{BN}^{[49]}$ or graphene ${ }^{[50]}$ and has a tunable gap depending on the doping concentration. The atomic models of 1-BCN and h-BCN used in our calculations are presented in Fig. 4(a). Based on the calculated phonon dispersions, there are no virtual frequencies for both 1-BCN and h-BCN, which indicates that they are stable materials.

Dirac cones of silicene in $\mathrm{Gr} / \mathrm{Si} / \mathrm{BN}$ are slightly opened because of the breaking of the space-inversion, as shown in Fig. 4(b). The Fermi level lies below the Dirac cones due to the presence of graphene. ${ }^{[38]}$ In both $1-\mathrm{BCN} / \mathrm{Si} / \mathrm{l}-\mathrm{BCN}$ and $\mathrm{h}-\mathrm{BCN} / \mathrm{Si} / \mathrm{h}-\mathrm{BCN}$, the Dirac cones are opened, with energy gaps of $19 \mathrm{meV}$ and $28 \mathrm{meV}$. In the absence of doping at $\mathrm{T}=0 \mathrm{~K}$, the Fermi level lies in the middle of the gap in 1-BCN/Si/l-BCN (Fig. 1(e)). While in h-BCN/Si/h-BCN, the Fermi level is below the opened Dirac cones (Fig. $1(f))$.

$\mathrm{HfO}_{2}$ is a large-gap semiconductor, like $\mathrm{BN}$. However, in $\mathrm{HfO}_{2} / \mathrm{Si} / \mathrm{HfO} 2$ (Fig. 4(e)), a gap of $131 \mathrm{meV}$ is opened as shown in Fig. 4(f). This gap may be caused by the interaction between silicon and oxygen. Therefore, $\mathrm{HfO}_{2} / \mathrm{Si} / \mathrm{HfO} 2$ are semiconducting. The binding energy of $\mathrm{HfO}_{2} / \mathrm{Si} / \mathrm{HfO}_{2}$ is $45 \mathrm{meV} / \AA^{2}$ which is larger than that of $\mathrm{BN} / \mathrm{Si} / \mathrm{BN}$, indicating the $\mathrm{HfO}_{2}$ layers have a stronger stabilization effect to silicene than BN.

\section{Conclusion}

In $\mathrm{BN} / \mathrm{Si} / \mathrm{BN}$ heterostructure with different rotation angles, lateral shifts, and limited strains, the electronic property of silicene is protected robustly. In $\mathrm{BN} / \mathrm{Si} / \mathrm{BN} / \mathrm{Si} / \mathrm{BN}$ heterostructure, the band structure is sensitive to the stacking configurations of the silicene layers due to interlayer coupling. The coupling can be inhibited by increasing the number of BN layers and becomes negligible at three BN layers. In BN/Si superlattices with silicene positioned in A-A stacking configuration, a 
conversion between Dirac lines and Dirac points can be achieved by changing the number of BN layers between silicene layers. Therefore, quasi-free-standing silicene can only be achieved in BN/Si superlattice when adjacent silicene layers are separated by at least three BN layers. Multi-conducting-channel BN/Si heterostructures is only possible when silicene layers are separated by no less than three BN layers. Besides, it is safer to use at least three BN layers on each side of silicene to protect the Dirac cones. At last, silicene sandwiched by $1-\mathrm{BCN}$ or $\mathrm{HfO}_{2}$ is semiconducting.

\section{Acknowledgements}

We thank Prof. Sokrates T. Pantelides in Vanderbilt University for providing advice on this project. Thanks to National Supercomputing Center in Tianjin for providing computational resources. 
Table 1. Silicene sandwiched by BN with different rotation angles and lattice mismatches.

\begin{tabular}{|c|c|c|c|c|c|}
\hline \multicolumn{2}{|c|}{$\mathrm{BN} / \mathrm{Si} / \mathrm{BN}$} & \multirow{2}{*}{$\begin{array}{l}\text { Rotation } \\
\text { angle }\end{array}$} & \multirow{2}{*}{$\begin{array}{l}\text { Lattice } \\
\text { mismatch }^{1}\end{array}$} & \multirow{2}{*}{$\begin{array}{l}\text { Binding } \\
\text { energy } \\
\mathrm{meV} / \AA^{2}\end{array}$} & \multirow{2}{*}{$\begin{array}{l}\text { Dirac cones } \\
\text { preserved } \\
(\mathrm{Y} / \mathrm{N})^{2}\end{array}$} \\
\hline Supercell & Top view & & & & \\
\hline $3 \mathrm{BN} / 2 \mathrm{Si} / 3 \mathrm{BN}$ & & $0^{\circ}$ & $2.3 \%$ & 32 & $\mathrm{Y}$ \\
\hline$\sqrt{ } 31 \mathrm{BN} / \sqrt{ } 13 \mathrm{Si} / \sqrt{ } 31 \mathrm{BN}$ & & $5^{\circ}$ & $-0.6 \%$ & 34 & $\mathrm{Y}$ \\
\hline$\sqrt{7} \mathrm{BN} / \sqrt{ } 3 \mathrm{Si} / \sqrt{ } 7 \mathrm{BN}$ & & $10.9^{\circ}$ & $0.5 \%$ & 33 & $\mathrm{Y}$ \\
\hline$\sqrt{3} 9 \mathrm{BN} / 4 \mathrm{Si} / \sqrt{ } 39 \mathrm{BN}$ & & $16.1^{\circ}$ & $-1.7 \%$ & 35 & $\mathrm{Y}$ \\
\hline $4 \mathrm{BN} / \sqrt{7} \mathrm{Si} / 4 \mathrm{BN}$ & & $19.1^{\circ}$ & $1.5 \%$ & 32 & $\mathrm{Y}$ \\
\hline
\end{tabular}

$1+(-)$ indicates tensile (compressive) strain in BN layers.

${ }^{2} \mathrm{Y}(\mathrm{N})$ indicates the Dirac cones of silicene are retained (destroyed). 
(a)

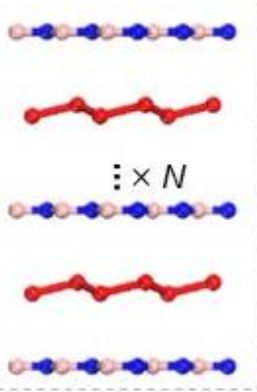

(d)

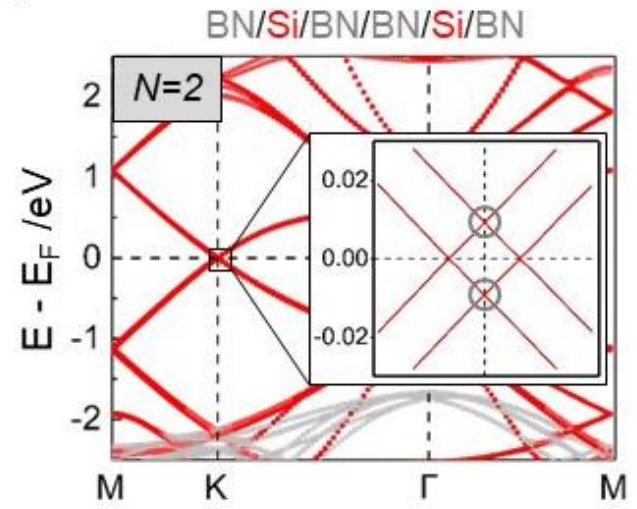

(b)

(c)

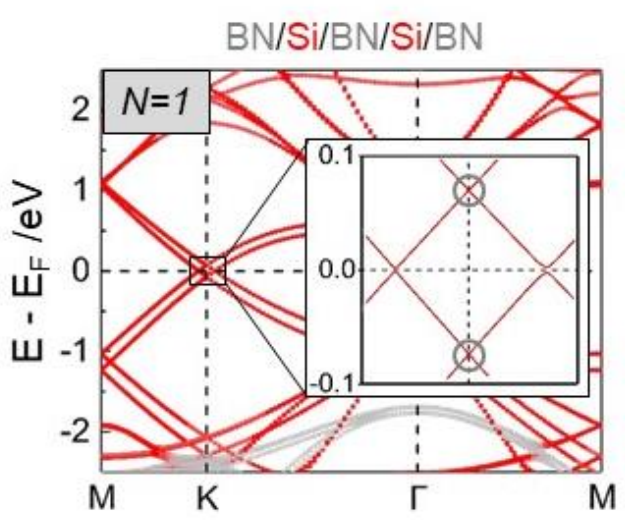

(e)

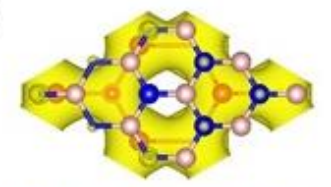

$8-\cos -\cos -0$

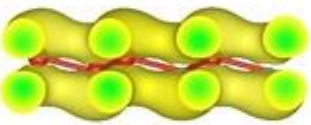

\&oक क्षिक्ष००

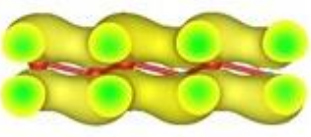

$-0-c-0200$

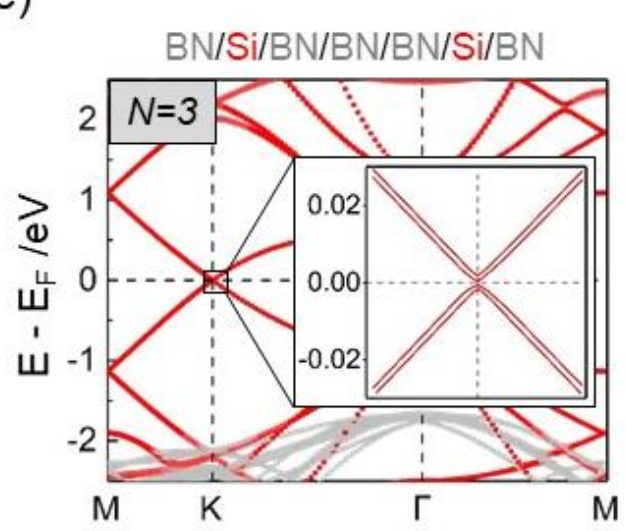

Fig. 1. (color online) Coupling between silicene layers intercalated by BN layers. (a): Supercell of a 2D vdWHs with two silicene layers encapsulated inside but separated by $N$ BN layers. (b): Energy band structure of supercell shown in (a) with $N=1$. (c): Partial charge density near the Fermi level $\left(\mathrm{E}_{\mathrm{f}} \pm 0.3 \mathrm{eV}\right)$ related to band structure shown in $\mathrm{b}$. (d)-(e): Energy band structures of the supercell shown in (a) with $N=2$ and 3, respectively. 
(a)
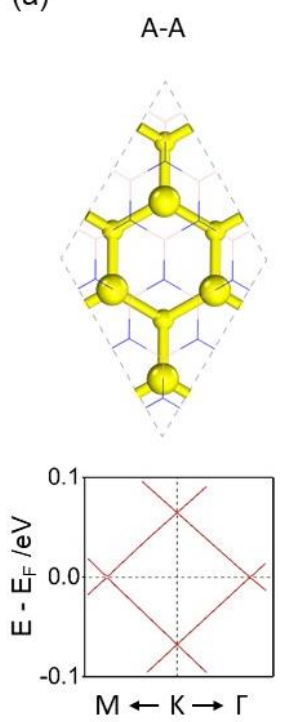

(b)

A-A mirror
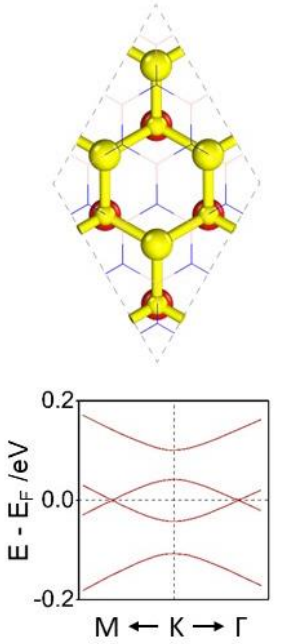

(c)
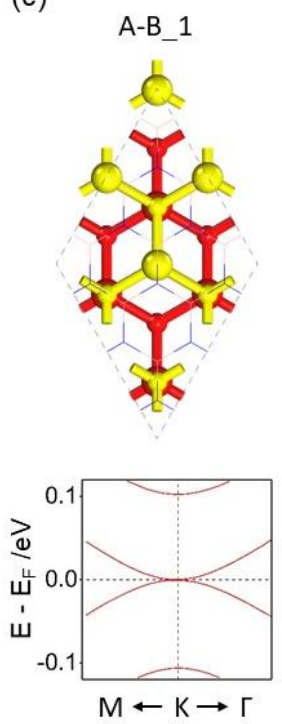

(d)

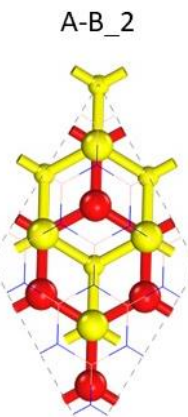

(e)
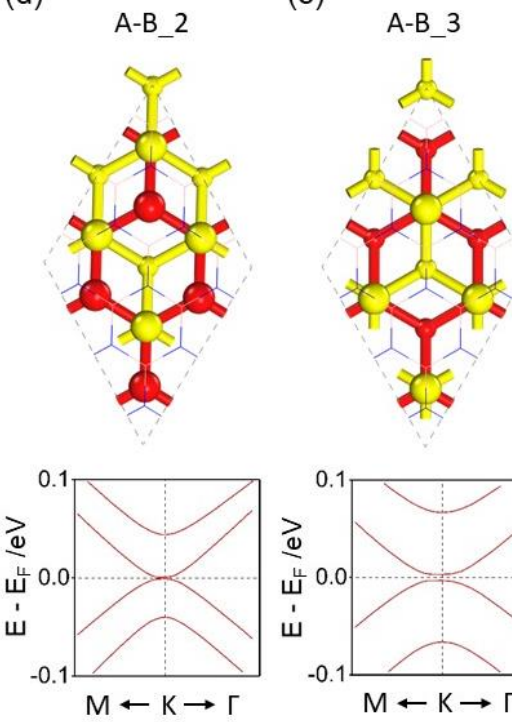

Fig. 2. (color online) Top views and energy band structures of $B N / S i / B N / S i / B N$ with different stacking configurations of silicene layers. To highlight the silicene layers, BN layers are shown in line style and the two silicene layers are colored by red and yellow respectively. The silicene layer colored in red is kept still and below. Two sub-lattices of silicene are indicated by the sizes of the balls that the larger balls represent the higher atoms in this top view. (a)-(b): The band structure of A-A stacking configuration. (c)(e): The band structure of A-B stacking configuration. 
(a)

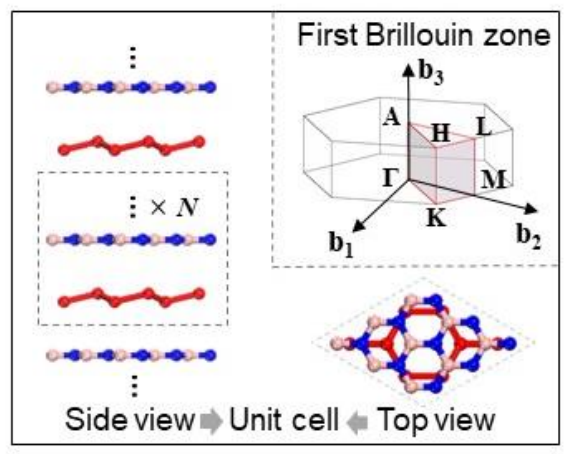

(c)

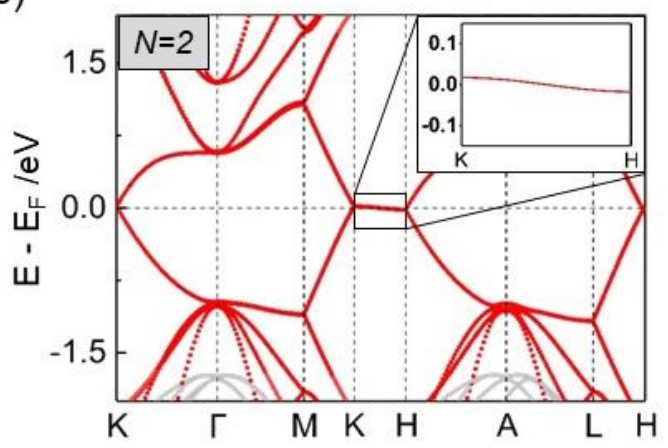

(b)

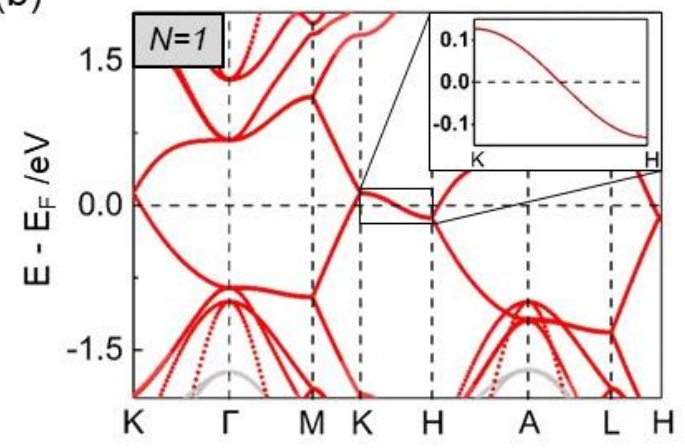

(d)

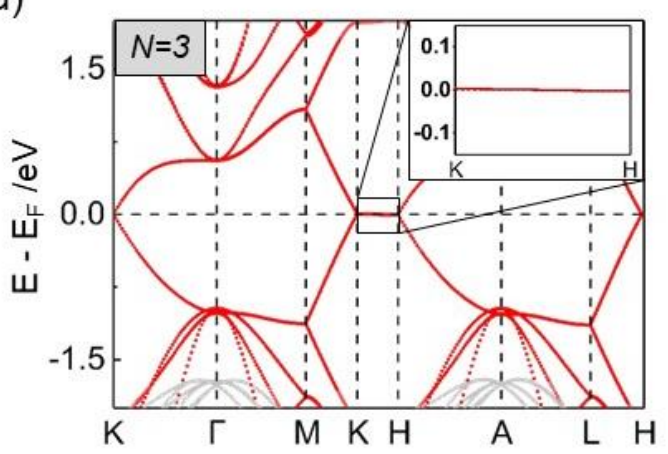

Fig. 3. (color online) Quasi-free-standing silicene in superlattices. (a): Unit cell and first Brillouin zone of the BN/silicene superlattices. (b)-(d): Energy band structures of the superlattices, differing from each other by the number of BN layers between adjacent silicene layers, from one to three. 
(a)

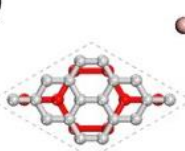

$\mathrm{Gr}, \mathrm{BN}, \mathrm{BCN}$

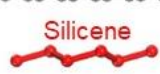

$0=00-00=00=00=0$ $\mathrm{Gr}, \mathrm{BN}, \mathrm{BCN}$

(b)

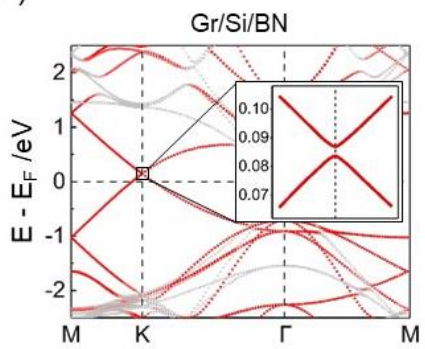

(c)
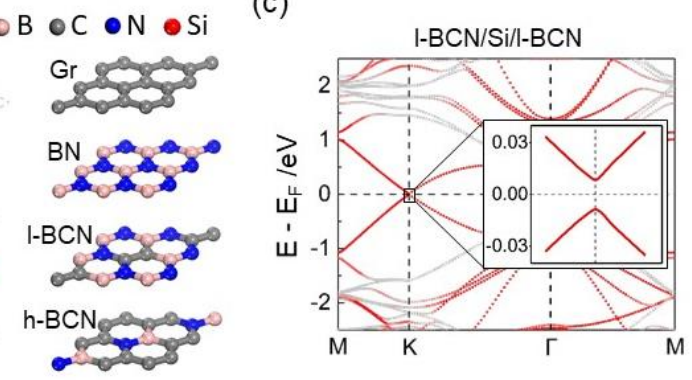

(d)

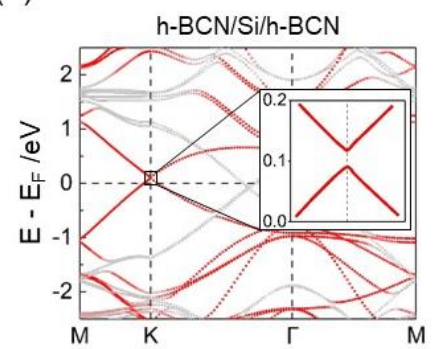

(e)

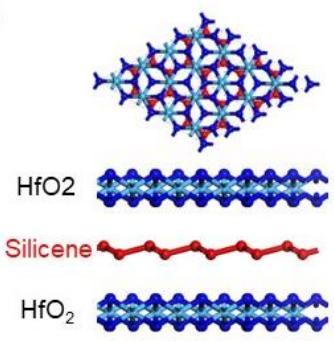

(f)

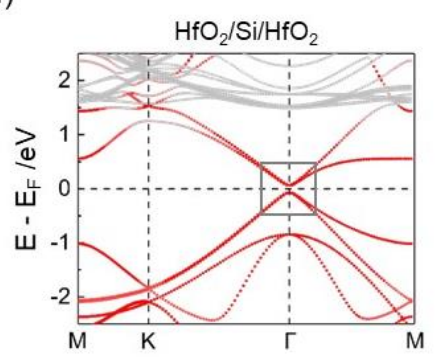

Fig. 4. (color online) Silicene sandwiched by other 2D materials where the Dirac cones are opened. (a): Left panel: Sandwich heterostructure with silicene in the middle. Right panel: Unit cells of cladding layers. (b)-(d): Projected energy band structures of $\mathrm{Gr} / \mathrm{Si} / \mathrm{BN}, \mathrm{BCN} / \mathrm{Si} / \mathrm{BCN}$ sandwich heterostructures. Contributions of silicene and cladding layers are quantitatively indicated by red-grey color scale, where a $100 \%$ contribution from silicene is marked by pure red. (e): Silicene sandwiched by $\mathrm{HfO}_{2}$. (f): The projected energy band structure of $\mathrm{HfO}_{2} / \mathrm{Si} / \mathrm{HfO}_{2}$. 


\section{References}

[1] Novoselov K S, Geim A K, Morozov S V, Jiang D, Zhang Y, Dubonos S V, Grigorieva I V, and Firsov A A 2004 Science 306666

[2] Zhang Y, Tan Y-W, Stormer H L, and Kim P 2005 Nature 438201

[3] Xu M, Liang T, Shi M, and Chen H 2013 Chem. Rev. 1133766

[4] Butler S Z, Hollen S M, Cao L, Cui Y, Gupta J A, Gutiérrez H R, Heinz T F, Hong S S, Huang J, Ismach A F, Johnston-Halperin E, Kuno M, Plashnitsa V V, Robinson R D, Ruoff R S, Salahuddin S, Shan J, Shi L, Spencer M G, Terrones M, Windl W, and Goldberger J E 2013 ACS Nano 72898

[5] Bhimanapati G R, Lin Z, Meunier V, Jung Y, Cha J, Das S, Xiao D, Son Y, Strano M S, Cooper V R, Liang L, Louie S G, Ringe E, Zhou W, Kim S S, Naik R R, Sumpter B G, Terrones H, Xia F, Wang Y, Zhu J, Akinwande D, Alem N, Schuller J A, Schaak R E, Terrones M, and Robinson J A 2015 ACS Nano 9 11509

[6] Naguib M, Mochalin V N, Barsoum M W, and Gogotsi Y 2014 Adv. Mater. 26 992

[7] Wang J, Deng S, Liu Z, and Liu Z 2015 Natl. Sci. Rev. 222

[8] Novoselov K S, Geim A K, Morozov S V, Jiang D, Katsnelson M I, Grigorieva I V, Dubonos S V, and Firsov A A 2005 Nature 438197

[9] Bolotin K I, Ghahari F, Shulman M D, Stormer H L, and Kim P 2009 Nature 462 196

[10] Du X, Skachko I, Duerr F, Luican A, and Andrei E Y 2009 Nature 462192

[11] Bolotin K I, Sikes K J, Jiang Z, Klima M, Fudenberg G, Hone J, Kim P, and Stormer H L 2008 Solid State Commun. 146351

[12] Castro Neto A H, Guinea F, Peres N M R, Novoselov K S, and Geim A K 2009 Rev. Mod. Phys. 81109

[13] Cahangirov S, Topsakal M, Aktürk E, Şahin H, and Ciraci S 2009 Phys. Rev. Lett. 102236804

[14] Şahin H, Cahangirov S, Topsakal M, Bekaroglu E, Akturk E, Senger R T, and Ciraci S 2009 Phys. Rev. B 80155453

[15] Li H, Hui-Xia F, and Meng S 2015 Chin.Phys.B 24086102

[16] Shao Z-G, Ye X-S, Yang L, and Wang C-L 2013 J. Appl. Phys. 114093712

[17] Ni Z, Liu Q, Tang K, Zheng J, Zhou J, Qin R, Gao Z, Yu D, and Lu J 2012 Nano Lett. 12113

[18] Scalise E, Houssa M, Cinquanta E, Grazianetti C, Broek B v d, Pourtois G, Stesmans A, Fanciulli M, and Molle A 2014 2D Materials 1011010

[19] Gao N, Li J C, and Jiang Q 2014 Phys. Chem. Chem. Phys. 1611673

[20] Du Y, Zhuang J, Liu H, Xu X, Eilers S, Wu K, Cheng P, Zhao J, Pi X, See K W, Peleckis G, Wang X, and Dou S X 2014 ACS Nano 810019

[21] Quhe R, Fei R, Liu Q, Zheng J, Li H, Xu C, Ni Z, Wang Y, Yu D, Gao Z, and Lu J 2012 Sci. Rep. 2853

[22] Drummond N D, Zólyomi V, and Fal'ko V I 2012 Phys. Rev. B 85075423

[23] Chun-Liang L, Ryuichi A, Kazuaki K, Noriyuki T, Emi M, Yousoo K, Noriaki T, and Maki K 2012 Appl. Phys. Express 5045802

[24] Feng B, Ding Z, Meng S, Yao Y, He X, Cheng P, Chen L, and Wu K 2012 Nano Lett. 123507

[25] Chen L, Liu C-C, Feng B, He X, Cheng P, Ding Z, Meng S, Yao Y, and Wu K 2012 Phys. Rev. Lett. 109056804

[26] Vogt P, De Padova P, Quaresima C, Avila J, Frantzeskakis E, Asensio M C, 
Resta A, Ealet B, and Le Lay G 2012 Phys. Rev. Lett. 108155501

[27] Meng L, Wang Y, Zhang L, Du S, Wu R, Li L, Zhang Y, Li G, Zhou H, Hofer W A, and Gao H-J 2013 Nano Lett. 13685

[28] Meng L, Wang Y-L, Zhang L-Z, Du S-X, and Gao H-J 2015 Chin.Phys.B 24 086803

[29] Huang L, Zhang Y-F, Zhang Y-Y, Xu W, Que Y, Li E, Pan J-B, Wang Y-L, Liu Y, Du S-X, Pantelides S T, and Gao H-J 2017 Nano Lett. 171161

[30] Fleurence A, Friedlein R, Ozaki T, Kawai H, Wang Y, and Yamada-Takamura Y 2012 Phys. Rev. Lett. 108245501

[31] Tao L, Cinquanta E, Chiappe D, Grazianetti C, Fanciulli M, Dubey M, Molle A, and Akinwande D 2015 Nature Nano. 10227

[32] Guo Z-X, Furuya S, Iwata J-i, and Oshiyama A 2013 Phys. Rev. B 87235435

[33] Wang Y-P and Cheng H-P 2013 Phys. Rev. B 87245430

[34] Arafune R, Lin C L, Nagao R, Kawai M, and Takagi N 2013 Phys. Rev. Lett. 110229701

[35] Lin C-L, Arafune R, Kawahara K, Kanno M, Tsukahara N, Minamitani E, Kim Y, Kawai M, and Takagi N 2013 Phys. Rev. Lett. 110076801

[36] Cahangirov S, Audiffred M, Tang P, Iacomino A, Duan W, Merino G, and Rubio A 2013 Phys. Rev. B $\mathbf{8 8} 035432$

[37] Zhong H-X, Quhe R-G, Wang Y-Y, Shi J-J, and Lü J 2015 Chin.Phys.B 24 087308

[38] Neek-Amal M, Sadeghi A, Berdiyorov G R, and Peeters F M 2013 Appl. Phys. Lett. 103261904

[39] Wang M, Liu L, Liu C-C, and Yao Y 2016 Phys. Rev. B 93155412

[40] Kou L, Ma Y, Yan B, Tan X, Chen C, and Smith S C 2015 ACS Appl. Mater. Interfaces 719226

[41] Smeu M, Zahid F, Ji W, Guo H, Jaidann M, and Abou-Rachid H 2011 J. Phys. Chem. C 11510985

[42] Kresse G and Furthmüller J 1996 Comp. Mat. Sci. 615

[43] Kresse G and Furthmüller J 1996 Phys. Rev. B 5411169

[44] Kresse G and Hafner J 1993 Phys. Rev. B 47558

[45] Kresse G and Joubert D 1999 Phys. Rev. B 591758

[46] Perdew J P, Burke K, and Ernzerhof M 1996 Phys. Rev. Lett. 773865

[47] Grimme S, Antony J, Ehrlich S, and Krieg H 2010 J. Chem. Phys. 132154104

[48] Kaloni T P, Tahir M, and Schwingenschlögl U 2013 Sci. Rep. 33192

[49] Huang C, Chen C, Zhang M, Lin L, Ye X, Lin S, Antonietti M, and Wang X 2015 Nature Communi. 67698

[50] Ba K, Jiang W, Cheng J, Bao J, Xuan N, Sun Y, Liu B, Xie A, Wu S, and Sun Z 2017 Sci. Rep. 745584 